\title{
Bioimaging based on antibody-conjugated amphiphilic polymer-core@shell quantum dots
}

1 Amir-Hassan Zarnani DMT, PhD

Professor, Reproductive Immunology Research Center, Avicenna Research Institute, ACECR, Tehran, Iran; Immunology Research Center, Iran University of Medical Sciences, Tehran, Iran

2 Mohammad-Reza Nejadmoghaddam PhD Assistant Professor, Nanobiotechnology Research Center, Avicenna Research Institute, ACECR, Tehran, Iran; Nanotechnology Research Center, Faculty of Pharmacy, Tehran University of Medical Sciences, Tehran, Iran

3 Mojtaba Mirhosseini Moghaddam PhD

Assistant Professor, Microinnova Engineering $\mathrm{GmbH}$, Allerheiligen bei Wildon, Austria
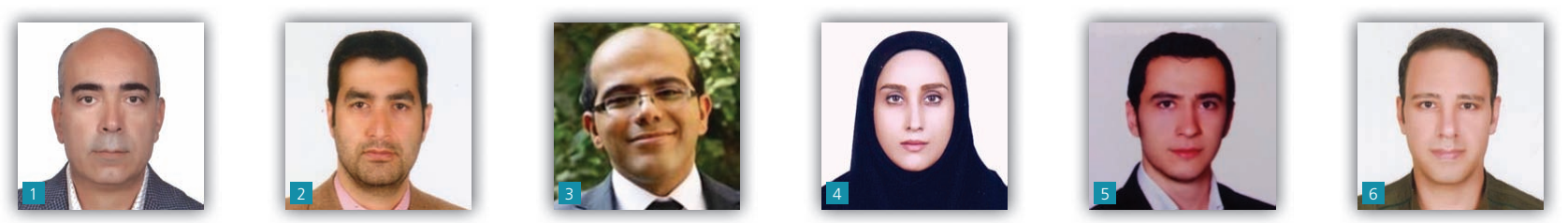

Quantum dots (QDs) are one of the most important achievements in the fast-growing nanotechnology field. Their unique optical properties make them superior to conventional fluorophores and essential in many applications. They have been successfully applied in various fields such as optics, electronics and biology. The ability to make QDs water soluble and use them to label specific biomolecules has led to promising applications such as high-resolution cellular imaging. Here, the authors present procedures for the synthesis and coating of cadmium selenide (CdSe) nanoparticles and their surface modification with an amphiphilic polymer for biological conjugation. The effectiveness of biological functionalization was assessed through cadmium selenide conjugation to an aminereactive anti-human CD4 monoclonal antibody. The results showed that the fabricated QD cores had a size distribution of about $2 \cdot 5-3 \mathrm{~nm}$ with the first exciton absorption peak and maximum emission wavelengths of 518 and $525 \mathrm{~nm}$, respectively. Coating with zinc sulfide (ZnS) markedly increased the emission intensity of QDs. Fourier transform infrared analysis confirmed surface modification of core@shell QDs with the polymer. Intense specific fluorescent signals were observed when the QD-conjugated antibody was electrophoresed. Taken together, the procedure introduced here could be viewed as a platform for functional and efficient synthesis of QD nanoparticles.

\section{Notation}

A absorbance

$b \quad$ path length: $\mathrm{cm}$

C concentration of a sample

c molar concentration of the attenuating species in the material

$P \quad$ radiant power leaving the sample

$P_{0} \quad$ radiant power passing through the sample cell

$T \quad$ transmittance

$\varepsilon \quad$ molar attenuation coefficient

\section{Introduction}

Research in nanomaterial sciences has received wide interest in the past two decades. During this period, amazing progress has been achieved with interdisciplinary applications in chemistry, physics, biology and medicine. ${ }^{1}$
Among nanostructures, semiconductor quantum dots (QDs), which are one particular type of inorganic nanocrystals, have been considered as a promising candidate for use in photodiodes/solar cells, ${ }^{2}$ lasers, light-emitting diodes ${ }^{3}$ phototransistors, biosensors and biological imaging. ${ }^{4-7}$ QDs are tiny light-emitting particles on the nanometer scale (approximately $2-10 \mathrm{~nm}$ ); these emissions depend on the size of the QDs. These nanoparticles are composed of groups II-VI or III-V atoms in the periodic table $\mathrm{e}^{8-10}$ and offer the opportunity to take advantage of size-induced changes to create new or improved materials for advanced photonic and phosphor applications. ${ }^{11}$ QDs are defined as a new class of fluorescent probes for both in vitro and in vivo biomolecular and cellular imaging. ${ }^{12,13}$

Fluorescence tagging of biomolecules, as a vital tool for developing immunoassays, cellular labeling and tissue imaging, is a commonly used approach in biological researches. In comparison 
with traditional fluorophores such as small organic dyes or fluorescent proteins, which usually have some weaknesses, including fixed emission spectra and liability to chemical changes and to photobleaching, ${ }^{14}$ QDs have excellent optical and photophysical properties encompassing size- and compositiontunable emission from visible (Vis) to infrared wavelengths, large absorption coefficients across a wide spectral range, simultaneous excitation of multiple fluorescence colors, chemical stability, very high levels of brightness and good photostability, which make them highly suitable in biological reesearch. ${ }^{10,12,15,16}$

Many studies on the synthesis of QDs such as cadmium sulfide $(\mathrm{CdS})$, cadmium selenide (CdSe) and cadmium telluride (CdTe) have been reported. ${ }^{17,18}$ Among the different QD compositions, the authors are particularly interested in cadmium selenide QDs, one of the most successful QDs that are prepared through organic solvent synthesis techniques. Cadmium selenide nanocrystals capped with a zinc sulfide $(\mathrm{ZnS})$ shell have enhanced luminescence intensity, stronger stability and exhibit a longer lifetime compared to uncapped ones. ${ }^{19}$ The shell (i.e. zinc sulfide) protects the core from oxidation, enhances the quantum yield $(\mathrm{QY})$ and prohibits cadmium ion $\left(\mathrm{Cd}^{2+}\right)$ release. ${ }^{20}$

For many biological applications of QDs, it is necessary for QDs to be transferred into aqueous solutions under physiological conditions. For this purpose, additional surface coating of QDs with an aqueous biocompatible organic layer is mandatory. ${ }^{19,21}$ There are two main approaches to QD surface modification, namely, (a) hydrophobic interaction of amphiphilic molecules with QDs and (b) interaction of polar groups of the coating molecules with the QD surface (the capping ligand exchange). Hydrophobic interaction of molecules seems to be one of the most basic and successful approaches, as numerous reports in literature supports. ${ }^{22}$

The present study synthesized cadmium selenide@zinc sulfide QDs and have developed a method for labeling them to an aminereactive biomolecule. The biomolecule-labeled QDs were further used to assess the authors' QD functionalization method through sodium dodecyl sulfate polyacrylamide gel electrophoresis (SDS-Page).

\section{Experimental section}

\subsection{General material}

Trioctylphosphine (TOP), trioctylphosphine oxide (TOPO), hexamethyldisilazane (HMDS), cadmium oxide (cadmium oxide), octadecylphosphonic acid (ODPA), dodecylamine, diethylzinc $\left(\mathrm{C}_{4} \mathrm{H}_{10} \mathrm{Zn} ; \mathrm{Et}_{2} \mathrm{Zn}\right)$, and selenium (Se) powder were purchased from various vendors. All solvents were obtained from commercial sources, and they were degassed, stored in a glove box and used without any further purification. All syntheses and purifications of nanocrystals prior to polymer coating were carried out under argon (Ar) flow. Deionized water was used in this study. Poly(isobutylene-alt-maleic anhydride) was bought from Sigma-Aldrich.

\subsection{Characterization}

Refluxing for the synthesis of cadmium selenide QDs was performed in a $100 \mathrm{ml}$ triple-neck round-bottomed borosilicate glass flask. Infrared spectra (Fourier transform infrared (FT-IR)) were collected from films of dried solution on potassium bromide $(\mathrm{KBr})$ crystals using a PerkinElmer 781 spectrophotometer and an Impact 400 Nicolet spectrophotometer.

The crystalline phase of the prepared QDs was characterized by X-ray diffraction (XRD). XRD studies on thin films of the nanoparticle reaction mixtures were carried out using a Panalytical X'Pert Pro instrument in the transmission mode with copper $(\mathrm{Cu}) \mathrm{K} \alpha$ radiation and with $\lambda=1.54 \AA$. Transmission electron microscopy (TEM) images of previously air-dried QDs were recorded with a Jeol Jem-2100UHR system, operated at $200 \mathrm{kV}$. Ultraviolet (UV)-Vis absorption spectra were obtained using a PerkinElmer Precisely Lambda 25 spectrophotometer at a wavelength of 300-700 nm. The as-prepared sample was directly used for optical characterization at room temperature under ambient conditions without any postpreparative treatment process. The fluorescence emission spectrum of the modified QDs was measured by using a PerkinElmer LS-45 system. A PI-ID 2000 syringe pump (Harvard Instruments) was used for delivery of precursors into a flask.

\subsection{Synthesis of cadmium selenide QDs}

The cadmium selenide core nanoparticles were prepared using a modification of a previously reported procedures (Scheme 1). ${ }^{23,24}$ In synthesis of cadmium selenide dots with different sizes, the injection temperature and the reaction time are important parameters. Heating of the system is accomplished using a mantle connected to a temperature controller. The nanoparticles can be prepared by reacting precursors at high temperatures in a coordinating solvent mixture. The details of a typical reaction are as follows: cadmium oxide $(0.03 \mathrm{~g})$, ODPA $(0.14 \mathrm{~g})$ and TOPO $(1.5 \mathrm{~g})$ were combined with stirring and degassed in a three-necked round-bottomed flask and heated to $150^{\circ} \mathrm{C}$ at a rate of $10^{\circ} \mathrm{C} / \mathrm{min}$ under vacuum $(<1$ Torr). The resulting mixture was heated at $150^{\circ} \mathrm{C}$ for $1 \mathrm{~h}$ under vacuum to remove residual water and vigorously stirred to melt all components except cadmium oxide. Then, the system was backfilled with argon and the temperature was increased to $380^{\circ} \mathrm{C}$ at a rate of $25^{\circ} \mathrm{C} / \mathrm{min}$ under argon flow until formation of the cadmium (Cd)/ODPA complex occurred, as indicated by a color change from an orange mixture to a clear and colorless solution. Afterward, $0.9 \mathrm{ml}$ of TOP was injected at $380^{\circ} \mathrm{C}$ and the mixture was kept at this temperature for $10 \mathrm{~min}$. The selenium solution was prepared by adding selenium powder $(0.029 \mathrm{~g})$ to $0.22 \mathrm{ml}$ of TOP in the glove box; the flask was sealed with a septum and then briefly stirred at room temperature until selenium was dissolved completely. This solution was then loaded into a syringe and injected rapidly into the reaction flask at $380^{\circ} \mathrm{C}$, causing an immediate drop in the temperature. Instantly, the heating mantle was removed and the reaction mixture was rapidly cooled to $25^{\circ} \mathrm{C}$ using compressed air across the surface of the flask. The color slowly changed from yellow to orange as 


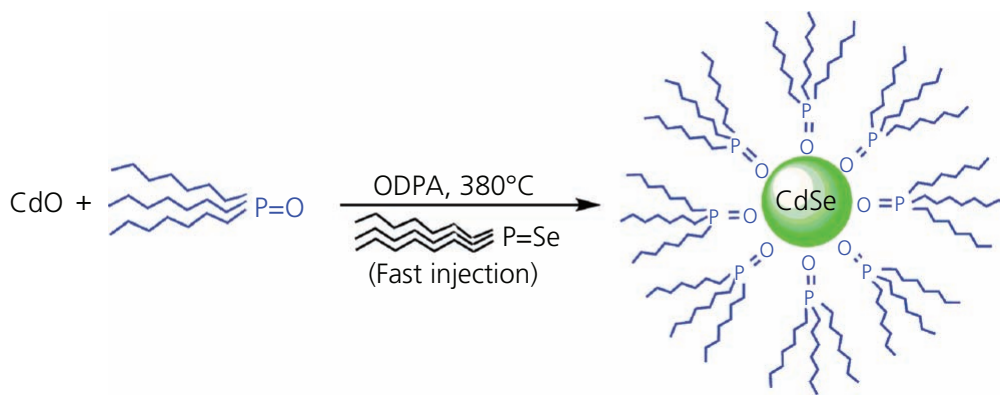

Scheme 1. Synthesis of cadmium selenide nanoparticles through the hot injection method

nucleation and growth of the nanoclusters occurred. At the end of the reaction, $3 \mathrm{ml}$ of toluene was added to the crude reaction mixture, which resulted in a clear solution, and then $10 \mathrm{ml}$ of pure methanol was added to this mixture, to precipitate nanocrystals. The precipitated nanoparticles were then collected by centrifugation (10 min at 10000 revolutions per minute (rpm)) and dried under an argon stream, producing $0.08 \mathrm{~g}$ of solid material.

\subsection{Coating of cadmium selenide core with zinc sulfide shell}

The encapsulation of the cadmium selenide QDs with a zinc sulfide shell was done using previously published procedures (Scheme 2). ${ }^{25-28}$ For zinc sulfide shell growth, $4 \mathrm{~g}$ of TOPO was added to a $50 \mathrm{ml}$ three-necked flask and heated up at a rate of $5^{\circ} \mathrm{C} / \mathrm{min}$ to $120^{\circ} \mathrm{C}$ under vacuum $(<1$ Torr). The flask was put under vacuum at $120^{\circ} \mathrm{C}$ for $20 \mathrm{~min}$, and then $0.3 \mathrm{ml}$ of TOP was injected into the flask. The cadmium selenide solution was prepared by dissolving $20 \mathrm{mg}$ of dry cadmium selenide nanocrystal powder in $2 \mathrm{ml}$ of toluene, and the resulting solution was injected into the flask. The solvent was removed by evacuating the flask under vacuum for $10 \mathrm{~min}$. The temperature of the mixture was then raised to $160^{\circ} \mathrm{C}$ at a rate of $3^{\circ} \mathrm{C} / \mathrm{min}$. The stock solution for zinc sulfide growth was freshly prepared by dissolving $0.261 \mathrm{ml}$ of $\mathrm{Et}_{2} \mathrm{Zn}(1.0 \mathrm{M}$ solution in heptanes) and $0.085 \mathrm{ml}$ of hexamethyldisilathiane $\left(\mathrm{C}_{6} \mathrm{H}_{18} \mathrm{Si}_{2} \mathrm{~S}\right)$ in $1.6 \mathrm{ml}$ of TOP under argon flow. A syringe pump was then used to deliver the zinc sulfide precursor solution to form a uniform shell growth and to prevent the nucleation of zinc sulfide crystals. After completion of the injections, the solution was cooled to $60^{\circ} \mathrm{C}$ and $1 \mathrm{ml}$ of butanol was added to avoid solidification and to quench the residual unreacted chemicals at this temperature. The solution was then transferred into a vial and stored for the polymer coating experiments. At the end of the reaction, $2 \mathrm{ml}$ of toluene was added to the crude reaction mixture, forming a clear solution, and then $5 \mathrm{ml}$ of pure methanol was added to this mixture, causing immediate precipitation. The solid was then collected by centrifugation (10 min at $10000 \mathrm{rpm})$ and dried under an argon stream. Decantation and evaporation of the supernatant to dryness produced $0.025 \mathrm{~g}$ of solid material. Finally, this product was dissolved in $5 \mathrm{ml}$ dry toluene, and the vials were sealed to protect them from light and air. The resulting core@shell nanocrystals can be stored at room temperature for several months. All semiconductor nanocrystals used for this work were synthesized by Stefan Kudera; the synthesis protocol has already been published. ${ }^{27}$

\subsection{Determination of the core@shell concentration}

In the polymer coating of cadmium selenide@zinc sulfide, the concentration of QDs was determined by comparison of their absorbance with a standard curve. Therefore, the concentration $C$ of a sample can be calculated from the Beer-Lambert law.

The Beer-Lambert law states that

1. $\%$ transmittance, $\% T=100 P / P_{0}=\mathrm{e}^{-\varepsilon b c}$

2. $T=P / P_{0}$

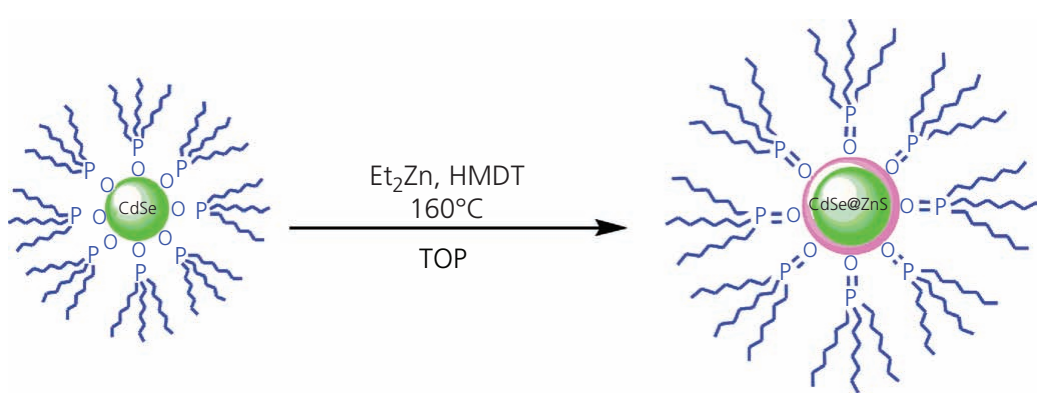

Scheme 2. Coating process for the synthesis of cadmium selenide@zinc sulfide QDs. HMDT, hexamethyldisilathiane 
Bioimaging based on antibodyconjugated amphiphilic polymercore@shell quantum dots

Zarnani, Nejadmoghaddam, Moghaddam et al.
3. $\% T=100 T$

4. absorbance, $A=\log _{10} P_{0} / P$

5. $A=\log _{10} 1 / T$

6. $A=\log _{10} 100 / \% T$

7. $A=2-\log _{10} \% T$

or equivalently that

8. $A=\varepsilon b c$

where $b$ is the path length in centimeters, $\varepsilon$ is the molar attenuation coefficient and $c$ is the molar concentration of the attenuating species in the material in moles per cubic decimeters or molar. $\varepsilon$ will be expressed in cubic decimeters per mole-centimeter or molar per centimeter, which is a commonly used unit. The Système International unit of $\varepsilon$ is square meters per mole.

\subsection{Synthesis of the amphiphilic polymer}

The polymer molecule for the coating was poly(maleic anhydridealt-1-tetradecene) $\left(\mathrm{C}_{18} \mathrm{H}_{30} \mathrm{O}_{3}\right.$; Pmat). The molecular weight (MW) of the polymer is about $9000 \mathrm{Da}$; it consists of approx 30 monomer units of $294.4 \mathrm{Da}$ MW. In its dry and solid form, the anhydride rings are closed; the properties of the molecule are mainly determined by its hydrophobic side chains, and it is therefore well soluble in organic solvents. For the synthesis of the amphiphilic polymer, $3.084 \mathrm{~g}$ of poly(isobutylene-alt-maleic anhydride) was placed in a round-bottomed flask. All the solvents employed for the synthesis of the polymer were anhydrous, in order to avoid the presence of water in the medium, which would have caused subsequent opening of the anhydride rings. In another flask, $2.70 \mathrm{~g}$ ( $15 \mathrm{mmol})$ of dodecylamine was dissolved in $100 \mathrm{ml}$ of anhydrous tetrahydrofuran (THF). The solution was added as quickly as possible with stirring to the polymer powder. This mixture was sonicated for several seconds $(\sim 30 \mathrm{~s})$ and heated to $55-60^{\circ} \mathrm{C}$ (just below boiling point of THF) for $1 \mathrm{~h}$. Afterward, the solution was preconcentrated to about one-third of the original volume in a rotation-evaporation (rotavap) system under reduced pressure ( $p=200-150 \mathrm{mbar}$ ) in order to enhance the reaction between the polymer and the amine. Once the sample had been concentrated, it was left stirring overnight. Then, the solvent was slowly evaporated by using the rotavap system until the polymer was completely dried (pale yellow solid). Finally, the amphiphilic polymer was redissolved in $25 \mathrm{ml}$ anhydrous chloroform $\left(\mathrm{CHCl}_{3}\right)$ with a resultant concentration of $0 \cdot 8 \mathrm{M}$ monomers $(=20 \mathrm{mmol} / 25 \mathrm{ml})$.

\subsection{Polymer coating of QDs}

Polymer-coated QDs was synthesized according to the standard protocol (Scheme 3). ${ }^{29}$ Briefly, $15 \mu \mathrm{l}$ of prepared polymer Pmat was added to dry chloroform $(0 \cdot 8 \mathrm{M})$ in a round-bottomed flask. Then, $1 \mathrm{ml}$ of anhydrous chloroform and $840 \mu \mathrm{l}$ of $3.07 \mu \mathrm{M}$ of cadmium selenide@zinc sulfide QDs in anhydrous chloroform were placed in this flask. The flask was heated to $55-60^{\circ} \mathrm{C}$ for approximately $40 \mathrm{~s}$ in order to give more flexibility to the polymer chain and to allow for better coating. Then the mixture was stirred in the rotavap system for $15 \mathrm{~min}$. The solvent was removed by rotary evaporation, and when there was no more solvent, the pressure was decreased to $20 \mathrm{mbar}$ in order to dry the sample completely. Finally, 2-3 $\mathrm{ml}$ of a buffer solution was added to product and agitated, forming a clear solution. To prepare the mentioned buffer solution, $25 \mathrm{ml}$ of 0.5 molar boric acid was dissolved in $200 \mathrm{ml}$ of distilled water and then the $\mathrm{pH}$ was adjusted to 12 using $1 \mathrm{M}$ solution of sodium hydroxide. Finally, it was diluted to $250 \mathrm{ml}$ with distilled water.
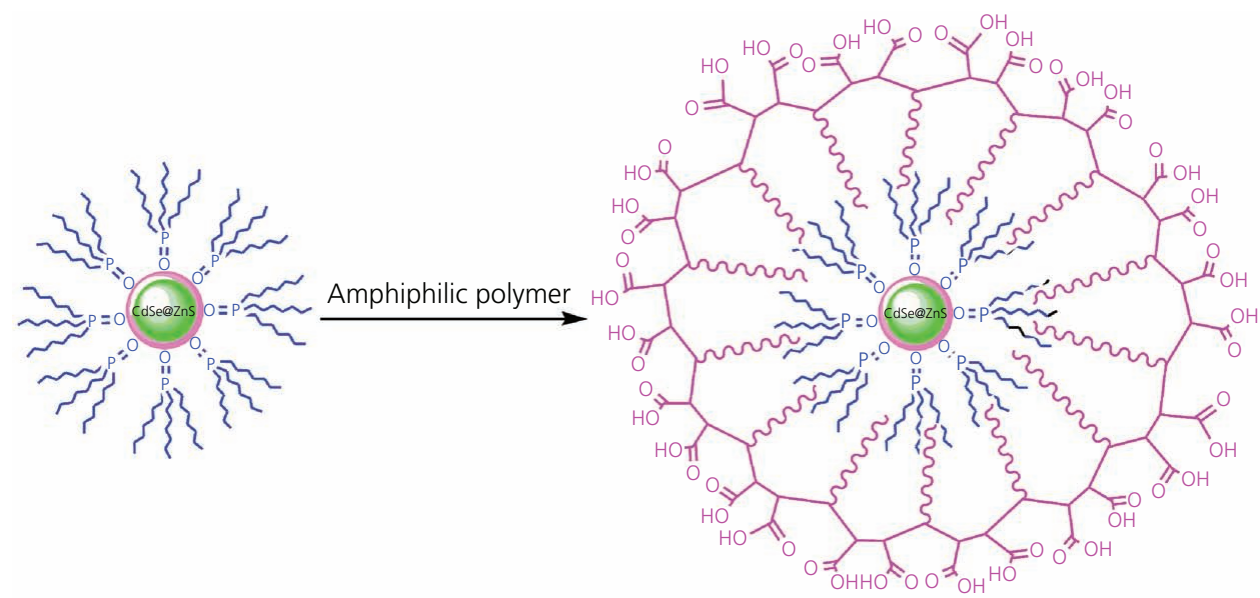

Scheme 3. Scheme of the phase-transfer procedure 
Bioimaging based on antibodyconjugated amphiphilic polymercore@shell quantum dots Zarnani, Nejadmoghaddam, Moghaddam et al.

\subsection{Preparation of biomolecule-QD conjugate}

In order to test the utility of the synthesized QD525 in biological applications, functionalized QD525 was conjugated to purified antihuman CD4 monoclonal antibody (mAb) (Sina Biotech, Tehran, Iran). To this end, carboxyl groups on the surface of the QDs were covalently bound to the amino groups of the antibody through the classical carbodiimide hydrochloride (EDC) coupling reaction. In brief, the QDs were first activated by dissolving $20 \mathrm{mM}$ EDC (Sigma-Aldrich) and $50 \mathrm{mM} N$-hydroxysuccinimide (SigmaAldrich) in $1 \mu \mathrm{M}$ QD solution ( $\mathrm{pH}=7 \cdot 4)$. Incubation was continued for $24 \mathrm{~h}$ at $4{ }^{\circ} \mathrm{C}$ with mixing using a magnetic stirrer to activate carboxyl groups. Then, an antibody was added to this mixture with an antibody:QD molar ratio of 4:1 for $2 \mathrm{~h}$ at room temperature.

\subsection{SDS-Page analysis}

Confirmation of antibody conjugation was performed by an SDSPage analysis of conjugated antibodies in order to visualize fluorescent signals under UV illumination. Briefly, purified unconjugated and QD-conjugated antibodies were boiled in sample buffer, containing $0.5 \mathrm{M}$ Tris-hydrochloric acid $(\mathrm{HCl})$ $(\mathrm{pH}=6 \cdot 8)$, SDS, bromophenol blue and glycerol, for $5 \mathrm{~min}$ and centrifuged. Clarified supernatants were loaded into a $12 \%$ SDS-Page gel and electrophoresed under non-reducing condition for $1.5 \mathrm{~h}$ at $50 \mathrm{~mA}$ and $100 \mathrm{~V}$. Fluorescent immunoglobulin bands were visualized under a UV transilluminator. In the next step, the gel was stained for $30 \mathrm{~min}$ with Coomassie Brilliant Blue R-250 (USB, USA) followed by destaining overnight.

\section{Results and discussion}

\subsection{Synthesis and characterization of cadmium selenide QDs}

Cadmium selenide QDs were prepared by using the hightemperature organic-phase method. ${ }^{24}$ These nanocrystals were synthesized in TOPO/TOP according to the proposed protocol; therefore, all the obtained products were treated in the same way to examine changes and were dissolved in toluene for UV-Vis spectroscopy, XRD characterization, photoluminescence (PL) spectroscopy, atomic force microscopy (AFM) and TEM.

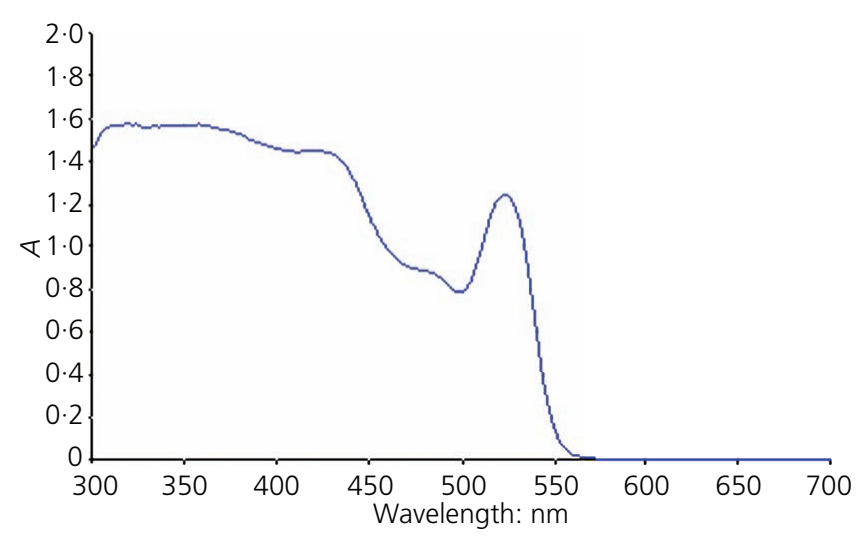

Figure 1. UV-Vis absorption spectra of cadmium selenide QDs
During the synthesis, aliquots were taken out at different time intervals, and UV-Vis spectra were recorded for each aliquot. The $\mathrm{UV}-$ Vis spectra are shown in Figure 1.

\subsubsection{UV-Vis analysis}

The spectroscopic properties of cadmium selenide QDs are shown in Figure 1. It is worth noting that cadmium selenide nanocrystals dispersed in toluene showed a narrow absorption peak or excitonic peak. As could be seen in Figure 1, there was an apparent absorption peak at $518 \mathrm{~nm}$ in the UV-Vis spectrum, indicating a narrow size distribution for the samples.

\subsubsection{Particle size evaluation}

The particle size obtained was about $2.5 \mathrm{~nm}$ based on comparison between an absorption peak in the UV-Vis spectrum and the standard diagram shown in Figure 2.

\subsubsection{PL spectroscopy}

The normalized PL spectrum showed a fairly symmetric shape, further proving the uniformity of the QDs. Figure 3 depicts the spectrum of cadmium selenide QDs, which has a narrow and sharp peak at approximately $525 \mathrm{~nm}$. The PL spectrum of the cadmium selenide QDs displays a uniform and homogeneous

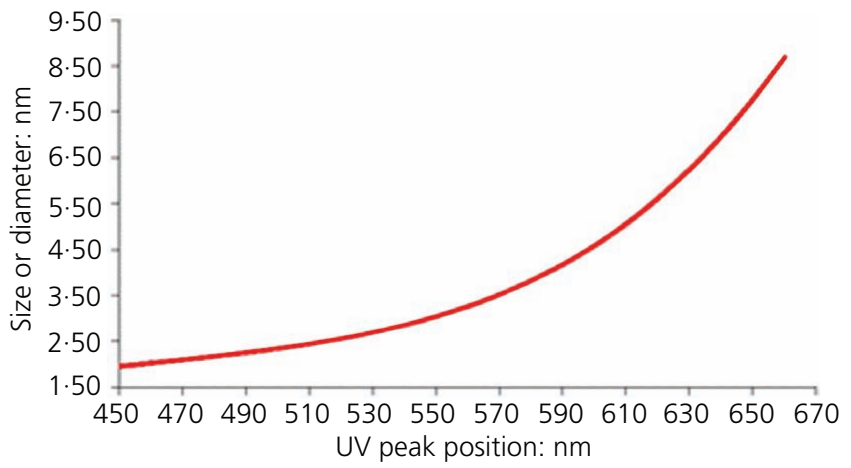

Figure 2. Standard sizing curve of cadmium selenide nanocrystals

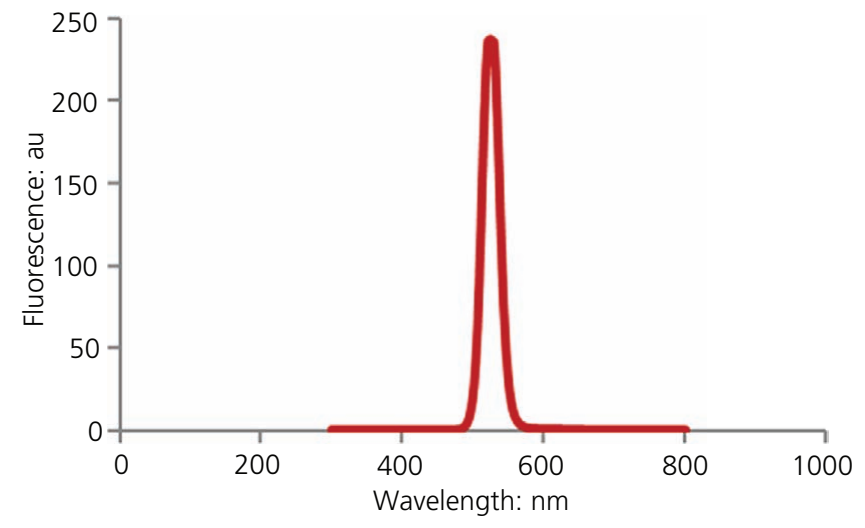

Figure 3. PL spectrum of the synthesized cadmium selenide QD sample 


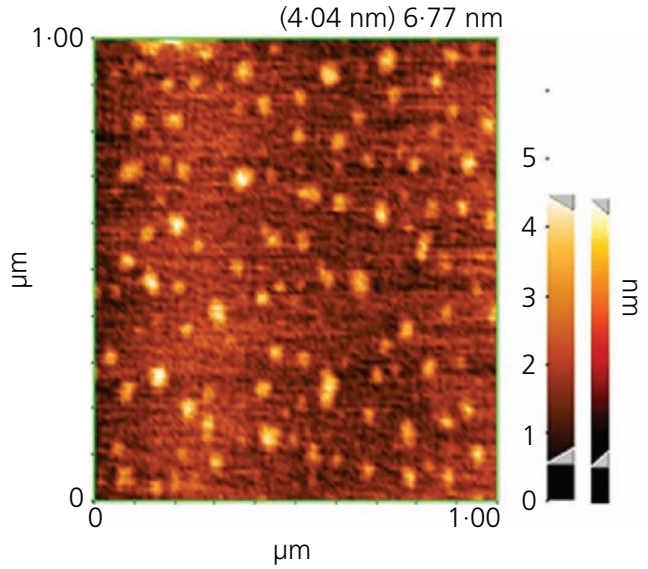

(a)

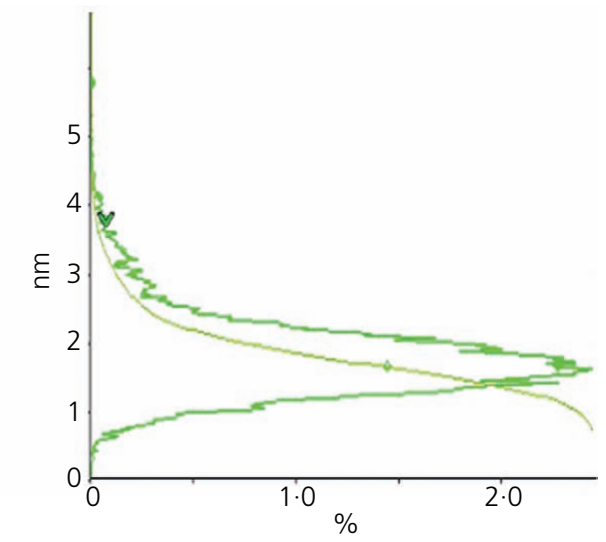

(b)

Figure 4. Topographic AFM images of cadmium selenide sample: (a) distribution network of the synthesized QD particles; (b) surface height distribution of the cadmium selenide QDs

distribution of these nanoparticles. The full width at less than half of the maximum of the PL spectrum was about $40 \mathrm{~nm}$, which illustrates the narrow size distribution of the prepared QDs.

\subsubsection{Topographic AFM images}

The AFM technique is carried out on cadmium selenide nanoparticle samples at atmospheric pressure and room temperature. The AFM technique provides three-dimensional images indicating the surface roughness, grain size, step height and pitch of cadmium selenide QD sample surface topography and enables viewing the QDs' distribution at a resolution similar to that of TEM. The results indicated a similar size distribution.

Figure 4(a) depicts the QD particle distribution network at the surface of the synthesized sample, while Figure 4(b) illustrates the surface roughness profile of the cadmium selenide QD sample. Figure $4(\mathrm{~b})$ is an illustration showing the surface height distribution of the synthesized cadmium selenide QDs.

\subsubsection{XRD characterization}

X-ray powder diffraction can provide useful information about the crystal structure of cadmium selenide QDs. XRD analysis exhibited the crystalline phase transformation at annealing temperatures of $350-450^{\circ} \mathrm{C}$ from cubic zinc blende. Figure 5 presents the XRD pattern obtained from powdered precipitate of cadmium selenide QDs used in this work. The XRD pattern of nanocrystalline cadmium selenide corresponds to the Miller indices (101), (110) and (112). The average crystalline size of the cadmium selenide QDs is estimated to be $3 \mathrm{~nm}$, which is very close to the results from the UV-Vis spectroscopy observation.

\subsection{Synthesis of cadmium selenide@zinc sulfide QDs and characterization}

The authors have demonstrated that it is possible to synthesize cadmium selenide@zinc sulfide QDs using a TOP/TOPO solvent.

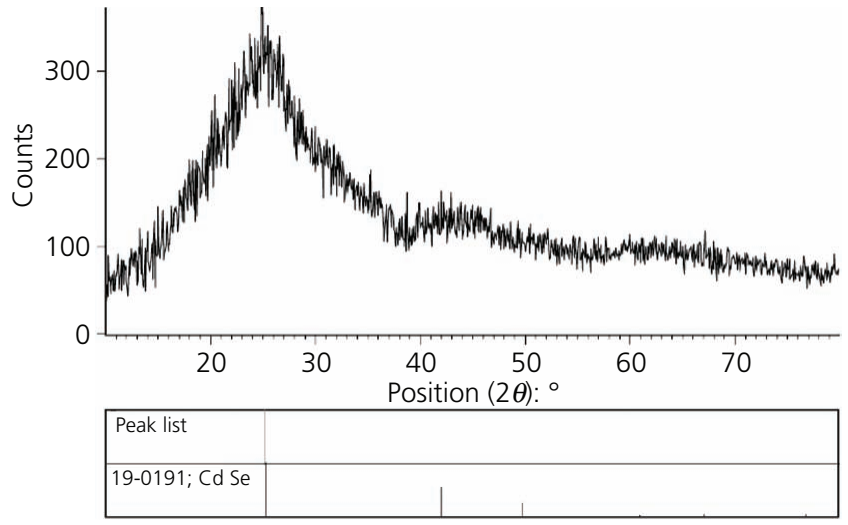

Figure 5. XRD pattern of the cadmium selenide QDs

To achieve good reproducibility of the synthesis of core@shell nanocrystals, special attention was paid to optimization of each preparation stage. Moreover, overcoating of QD core prevents the leakage of core materials (cadmium ion), thereby reducing the toxicity of QDs. The luminescence QY also decreases with increase in shell thickness, limiting their use of QDs optoelectronic devices. These results clearly indicate the need of optimizing the shell thickness of the overcoating layer. A few attempts have been made to follow the overcoating process using $\mathrm{UV}-\mathrm{Vis}$ analysis, and these aspects are summarized later.

The characterization data used in all of the experiments reported in this study are detailed in the following. The results of TEM, UV-Vis spectroscopy and PL studies reveal the size, composition and optical properties of cadmium selenide@zinc sulfide QDs.

The zinc sulfide-overcoated cadmium selenide QDs were characterized by UV-Vis absorption spectroscopy, PL spectroscopy and TEM, and the results are discussed. 
Bioimaging based on antibodyconjugated amphiphilic polymercore@shell quantum dots Zarnani, Nejadmoghaddam, Moghaddam et al.

\subsubsection{UV-Vis analysis of cadmium selenide@zinc sulfide QDs}

The UV-Vis absorption spectrum was measured at room temperature. Chemically synthesized cadmium selenide@zinc sulfide QDs exhibit nearly monodispersive size distribution, and the controllable optical properties are a function of their size. The UV-Vis spectrum shows that the absorption peak of these QDs in the toluene solution is $\sim 521 \mathrm{~nm}$ (Figure 6), indicating that the synthesis of cadmium selenide@zinc sulfide QDs was successful.

\subsubsection{PL analysis of cadmium selenide@zinc sulfide QDs}

In Figure 7, the PL spectrum of the colloidal cadmium selenide@zinc sulfide QDs without surface modification shows a distinct narrow emission peak at $525 \mathrm{~nm}$. This peak can be attributed to cadmium selenide core nanocrystals, which are created with the addition of a zinc sulfide shell; the increase in intensity is a result of passivation of cadmium selenide nanocrystals with a zinc sulfide shell.

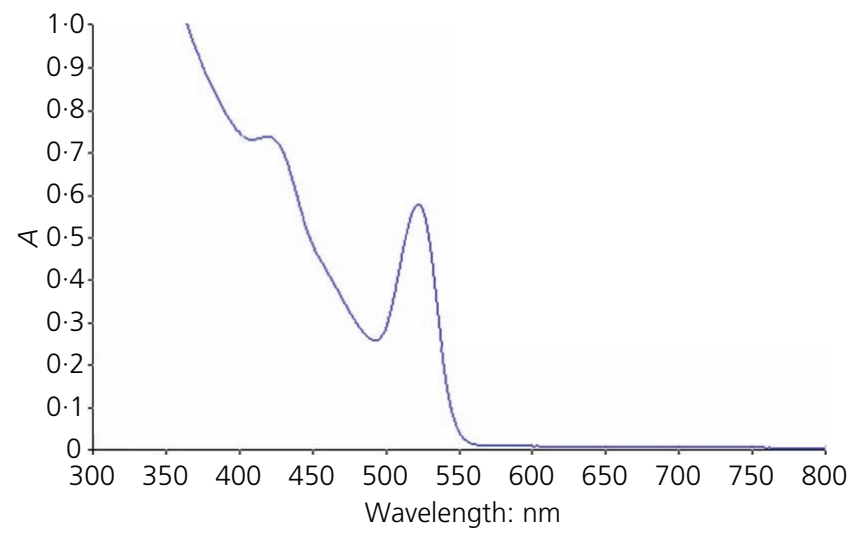

Figure 6. UV-Vis absorption spectrum of cadmium selenide@zinc sulfide QDs

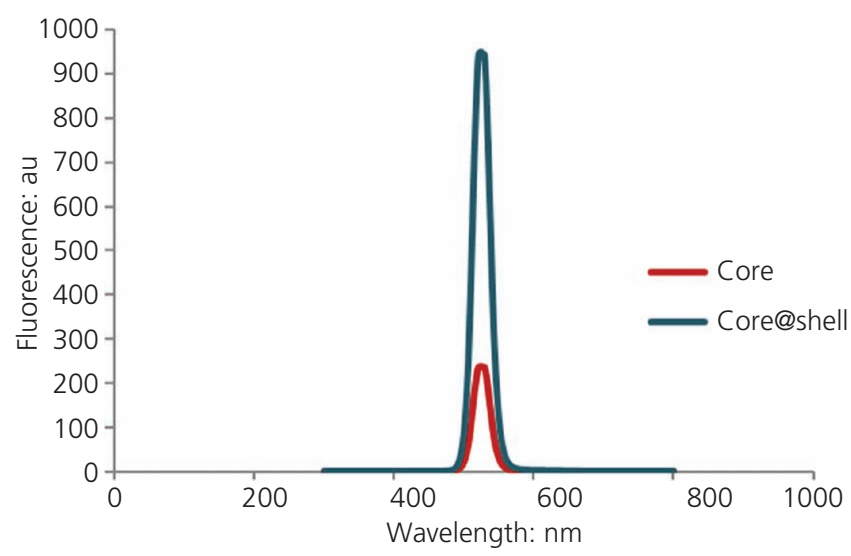

Figure 7. Normalized PL spectrum of the synthesized cadmium selenide QD and cadmium selenide@zinc sulfide samples

\subsubsection{TEM evaluation of cadmium selenide@zinc sulfide QDs}

TEM evaluation of the stock solution of cadmium selenide@zinc sulfide QDs revealed individual and monodispersive nanoparticles. Colloidal cadmium selenide@zinc sulfide QDs without surface modification have TOPO as hydrophobic surfactant.

The shape and size of cadmium selenide@zinc sulfide QDs were also observed by TEM. A typical size distribution, estimated from the TEM images, is shown in Figure 8.

\subsection{Surface modification of cadmium selenide@zinc sulfide QDs by polymer and their characterization}

This section describes a complete protocol for the synthesis and water solubilization of hydrophobic cadmium selenide@zinc sulfide QDs using designer amphiphilic polymeric coatings. The method is based on functionalization of an anhydride polymer backbone with nucleophilic agents. Small functional groups and polymeric chains can be integrated into the coating prior to solubilization.

Pmat is a copolymer with two alternative monomeric units. The monomeric unit of maleic anhydride can easily react with an amino or hydroxyl group to form one carboxylic group. Other monomeric units of hydrophobic dodecylamine groups have one hydrophobic 12-carbon alkane side chain that is very good for interacting with the side chain of TOPO on the surface of the QDs hydrophobically.

\subsubsection{FT-IR spectroscopy}

In this work, polymer-capped cadmium selenide@zinc sulfide QDs were studied by FT-IR spectroscopy in an effort to understand better the local environment of the polymer on the

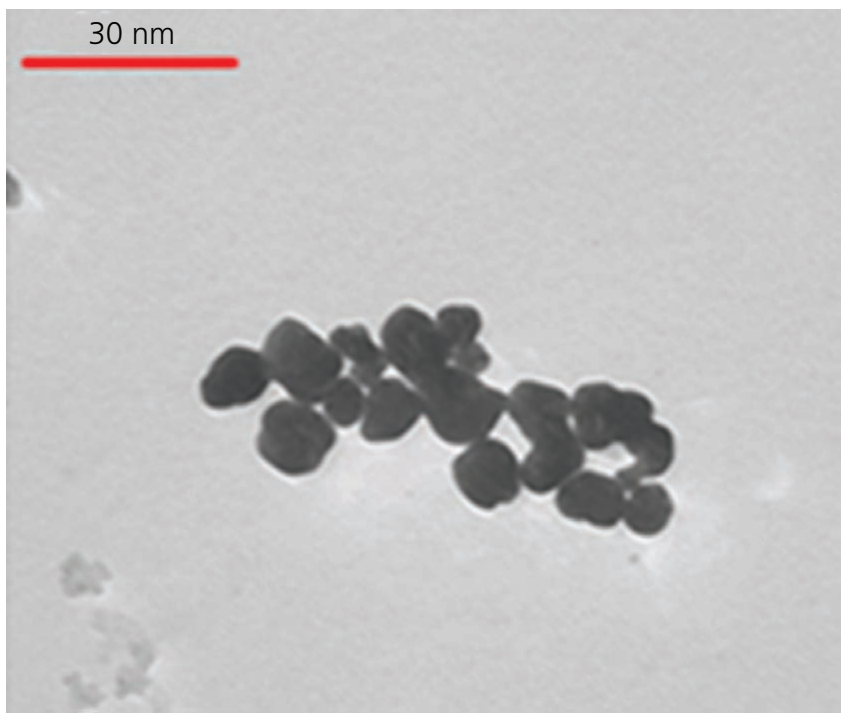

Figure 8. TEM image of zinc sulfide-overcoated cadmium selenide QDs 
QDs surface. Cadmium selenide@zinc sulfide QDs were encapsulated with Pmat polymers (TOPO-Pmat QDs) in chloroform using a solid dispersion method. The TOPO-Pmat coating is desirable for increasing aqueous solubility and ease of conjugation to targeting moieties. The resultant polymer (TOPO-Pmat)-coated cadmium selenide@zinc sulfide QDs were verified by FT-IR characterization (Figure 9). The FT-IR spectrum of polymer-coated QDs shows some peaks at 3292.49, 2925.71, $1865 \cdot 19$ and $1261.94 \mathrm{~cm}^{-1}$, which are attributed to $\mathrm{O}-\mathrm{H}, \mathrm{C}-\mathrm{H}$, $\mathrm{C}=\mathrm{O}$ and $\mathrm{C}-\mathrm{O}$ stretching vibrations, respectively, confirming the presence of carbonyl or carboxyl groups at the surface of QDs. The intense broad band at $3292.49 \mathrm{~cm}^{-1}$ is attributed to stretching of the $\mathrm{O}-\mathrm{H}$ bond of $\mathrm{COO}-\mathrm{H}$.

The strongest signals, observed at higher frequencies, are attributed to asymmetric $\left(2924 \mathrm{~cm}^{-1}\right)$ and symmetric $\left(2852 \mathrm{~cm}^{-1}\right)$ stretching of the methylene groups in the chain of the Pmat polymers. The intense band at $1865 \cdot 19 \mathrm{~cm}^{-1}$ is associated with stretching of the $\mathrm{C}=\mathrm{O}$ bond of carbonyl or carboxyl groups. The bands present at 1587 and $1261 \mathrm{~cm}^{-1}$ are attributed to bending vibrations of the $\mathrm{O}-\mathrm{H}$ and $\mathrm{C}-\mathrm{O}$ bonds in $\mathrm{CO}-\mathrm{H}$ groups, respectively.

\subsection{Characterization of QD-conjugated antibody}

SDS-Page electrophoresis was used as a simple and direct visual method for confirming QD conjugation to antibody. Expectedly, an unconjugated antibody showed a single band of about $150 \mathrm{kDa}$ (Figure 10), which was in good agreement with the MW of antibodies. In a conjugated fraction, a band with a higher MW was observed, indicating successful conjugation. Indeed, a faint band with an MW of $150 \mathrm{kDa}$ corresponded to unconjugated antibodies. The conjugated antibody produced a brilliant band with a MW above $150 \mathrm{kDa}$ under UV illumination, further confirming successful conjugation of the antibody with QD.

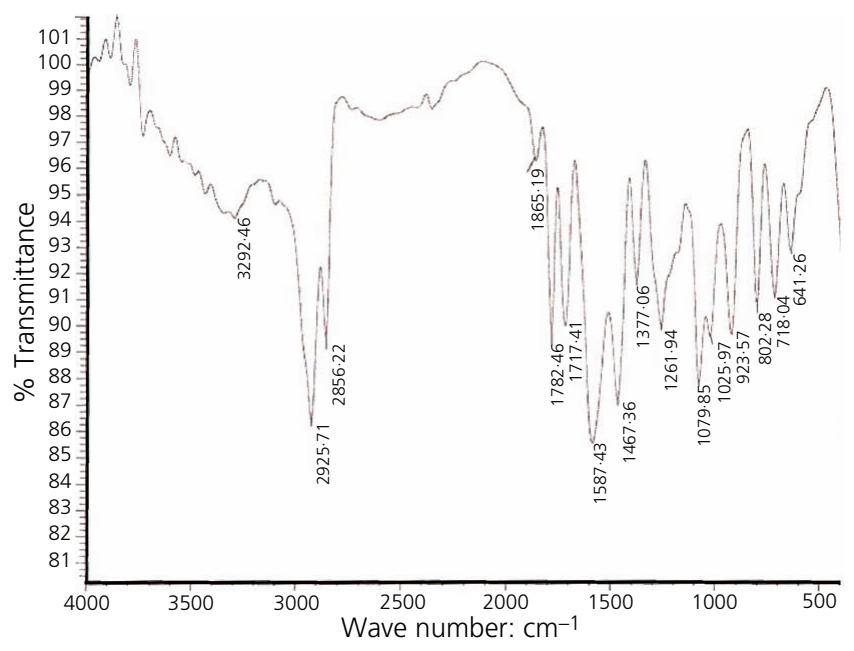

Figure 9. FT-IR spectrum of polymer-coated QDs in a potassium bromide pellet

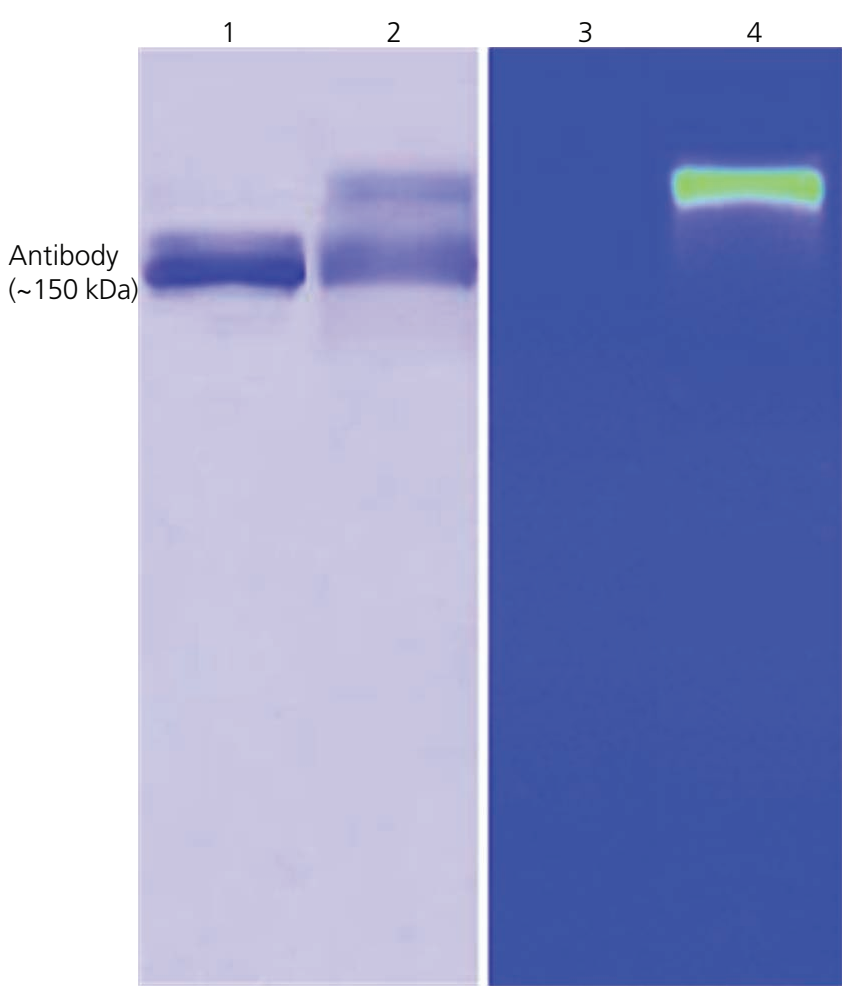

Figure 10. SDS-Page analysis of the QD525-conjugated antibody. The anti-human CD4 antibody was conjugated with QD525, and the efficacy of conjugation was confirmed with SDS-Page analysis. Lanes 1 and 3 show the unconjugated antibody with Coomassie Blue staining and UV illumination, respectively. Lanes 2 and 4 depict the conjugated antibody under conditions mentioned earlier

\section{Conclusion}

In conclusion, optically active cadmium selenide@zinc sulfide QDs were successfully synthesized and employed for labeling to amine-reactive biomolecules. During synthesis, the reaction condition was optimized to achieve a narrow size distribution with a sharp excitonic peak at $525 \mathrm{~nm}$. To have an insight into the capability of functionalized QDs to be coupled with biomolecules, the QDs were conjugated to anti-human CD4 mAb. QD surface functionalization with organic ligands as well as their extraction into aqueous media resulted in efficient coupling of cadmium selenide@zinc sulfide QDs with the antibody. The QD525conjugated antibody was further used to assess the authors' QD functionalization method through SDS-Page. This is the first time, to the authors' knowledge, that SDS-Page was used to confirm biofunctionalization of such nanoparticles. The conjugation method can be potentially used for biological applications of QDs. Obviously, for such applications, it is necessary that unconjugated fractions of the biomolecule must be removed by separation and purification methods such as gel filtration.

\section{Acknowledgements}

This work was supported by a grant from Avicenna Research Institute $^{30}$ and conducted in Nanobiotechnology Research Center, 
Emerging Materials Research

Volume 7 Issue EMR4
Bioimaging based on antibody-

conjugated amphiphilic polymer-

core@shell quantum dots

Zarnani, Nejadmoghaddam, Moghaddam et al.
Avicenna Research Institute. The authors would like to thank S. Najafzadeh, and F. Yazdi, for technical assistance.

\section{REFERENCES}

1. Roco MC, Mirkin CA and Hersam MC (2011) Nanotechnology research directions for societal needs in 2020: summary of international study. Journal of Nanoparticle Research 13(3): 897-919.

2. Van Sark WG, Frederix PL, Van den Heuvel DJ et al. (2001) Photooxidation and photobleaching of single CdSe/ZnS quantum dots probed by room-temperature time-resolved spectroscopy. Journal of Physical Chemistry B 105(35): 8281-8284.

3. Goldman ER, Medintz IL, Hayhurst A et al. (2005) Self-assembled luminescent $\mathrm{CdSe}-\mathrm{ZnS}$ quantum dot bioconjugates prepared using engineered poly-histidine terminated proteins. Analytica Chimica Acta 534(1): 63-67.

4. Smith AM, Duan H, Mohs AM and Nie S (2008) Bioconjugated quantum dots for in vivo molecular and cellular imaging. Advanced Drug Delivery Reviews 60(11): 1226-1240.

5. Law WC, Yong KT, Roy l et al. (2009) Aqueous phase synthesis of highly luminescent $\mathrm{CdTe} / \mathrm{ZnTe}$ core/shell quantum dots optimized for targeted bioimaging. Small 5(11): 1302-1310.

6. Wang P, Abrusci A, Wong HM et al. (2006) Photoinduced charge transfer and efficient solar energy conversion in a blend of a red polyfluorene copolymer with CdSe nanoparticles. Nano Letters 6(8): 1789-1793.

7. Zhou L, Gao C, Hu X and Xu W (2010) One-pot large-scale synthesis of robust ultrafine silica-hybridized CdTe quantum dots. ACS Applied Materials \& Interfaces 2(4): 1211-1219.

8. Leutwyler WK, Bürgi SL and Burgl H (1996) Semiconductor clusters, nanocrystals, and quantum dots. Science 271(5251): 933-937.

9. Dey SC and Nath SS (2012) Size-dependent fluorescence in CdSe quantum dots. Emerging Materials Research 1(3): 117-120.

10. Michalet X, Pinaud F, Lacoste TD et al. (2001) Properties of fluorescent semiconductor nanocrystals and their application to biological labeling. Single Molecules 2(4): 261-276.

11. Adem YT, Schwarz KA, Duenas E et al. (2014) Auristatin antibody drug conjugate physical instability and the role of drug payload. Bioconjugate Chemistry 25(4): 656-664.

12. Yong KT, Qian J, Roy l et al. (2007) Quantum rod bioconjugates as targeted probes for confocal and two-photon fluorescence imaging of cancer cells. Nano Letters 7(3): 761-765.

13. Jamieson T, Bakhshi R, Petrova D et al. (2007) Biological applications of quantum dots. Biomaterials 28(31): 4717-4732.

14. Frasco MF and Chaniotakis N (2009) Semiconductor quantum dots in chemical sensors and biosensors. Sensors 9(9): 7266-7286.
15. Xiong $\mathrm{H}$, Chen $\mathrm{ZH}$, Hyomi $\mathrm{K}$ et al. (2006) Coupling of quantum states in a single $\mathrm{CdSe} / \mathrm{ZnSe}$ quantum dot molecule. Journal of Luminescence 119: 193-197.

16. Dong W, Guo L, Wang M and Xu S (2009) CdTe QDs-based prostatespecific antigen probe for human prostate cancer cell imaging. Journal of Luminescence 129(9): 926-930.

17. Jacak L, Hawrylak P and Wójs A (2013) Quantum Dots. Springer, Berlin, Germany.

18. Baruah L and Nath SS (2013) Oleic acid-capped CdTe quantum dots and their applications as nano-LED. Emerging Materials Research 2(4): 186-190.

19. Liu LW, Hu SY, Pan Y et al. (2014) Optimizing the synthesis of $\mathrm{CdS} / \mathrm{ZnS}$ core/shell semiconductor nanocrystals for bioimaging applications. Beilstein Journal of Nanotechnology 5: 919-926.

20. Bilan R, Fleury F, Nabiev I and Sukhanova A (2015) Quantum dot surface chemistry and functionalization for cell targeting and imaging. Bioconjugate Chemistry 26(4): 609-624.

21. Frangioni JV (2003) In vivo near-infrared fluorescence imaging. Current Opinion in Chemical Biology 7(5): 626-634.

22. Scheerschmidt $K$ and Werner $P$ (2002) Characterization of structure and composition of quantum dots by transmission electron microscopy. In Nano-optoelectronics: Concepts, Physics and Devices (Grundmann M (ed.)). Springer, Berlin, Germany, pp. 67-98.

23. Foos EE, Wilkinson J, Mäkinen AJ et al. (2006) Synthesis and surface composition study of CdSe nanoclusters prepared using solvent systems containing primary, secondary, and tertiary amines. Chemistry of Materials 18(12): 2886-2894.

24. Carbone L, Nobile C, De Giorgi M et al. (2007) Synthesis and micrometer-scale assembly of colloidal $\mathrm{CdSe} / \mathrm{CdS}$ nanorods prepared by a seeded growth approach. Nano Letters 7(10): 2942-2950.

25. Cordero S, Carson P, Estabrook R, Strouse G and Buratto S (2000) Photo-activated luminescence of CdSe quantum dot monolayers. Journal of Physical Chemistry B 104(51): 12137-12142.

26. Sperling RA, Pellegrino T, Li JK, Chang WH and Parak WJ (2006) Electrophoretic separation of nanoparticles with a discrete number of functional groups. Advanced Functional Materials 16(7): 943-948.

27. Pellegrino T, Manna L, Kudera S et al. (2004) Hydrophobic nanocrystals coated with an amphiphilic polymer shell: a general route to water soluble nanocrystals. Nano Letters 4(4): 703-707.

28. Weaver J, Zakeri R, Aouadi S and Kohli P (2009) Synthesis and characterization of quantum dot-polymer composites. Journal of Materials Chemistry 19(20): 3198-3206.

29. Fernández-Argüelles MT, Yakovlev A, Sperling RA et al. (2007) Synthesis and characterization of polymer-coated quantum dots with integrated acceptor dyes as FRET-based nanoprobes. Nano Letters 7(9): 2613-2617.

30. Avicenna Research Institute (2018) http://www.ari.ir (accessed 15/10/2018).

\section{How can you contribute?}

To discuss this paper, please submit up to 500 words to the journal office at journals@ice.org.uk. Your contribution will be forwarded to the author(s) for a reply and, if considered appropriate by the editor-in-chief, it will be published as a discussion in a future issue of the journal.

ICE Science journals rely entirely on contributions from the field of materials science and engineering. Information about how to submit your paper online is available at www.icevirtuallibrary.com/page/authors, where you will also find detailed author guidelines. 